\title{
Chronic Atrophic Gastritis
}

National Cancer Institute

\section{Source}

National Cancer Institute. Chronic Atrophic Gastritis. NCI Thesaurus. Code C7405.

Atrophic gastritis that is persistent and long-standing. 